\title{
Addenda to the insect fauna of Al-Baha Province, Kingdom of Saudi Arabia with zoogeographical notes
}

\author{
Magdi S. El-Hawagrya,c, Mostafa R. Sharaf ${ }^{\mathrm{b}}$, Hathal M. Al Dhafer ${ }^{\mathrm{b}}$, Hassan H. Fadl ${ }^{\mathrm{b}}$ \\ and Abdulrahman S. Aldawood ${ }^{\mathrm{b}}$

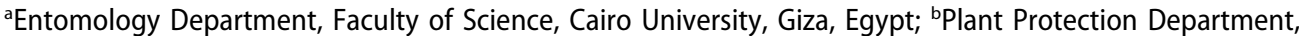 \\ College of Food and Agriculture Sciences, King Saud University, Riyadh, Kingdom of Saudi Arabia; 'Survey \\ and Classification of Agricultural and Medical Insects in Al-Baha Province, Al-Baha University, Al-Baha, \\ Saudi Arabia
}

\begin{abstract}
The first list of insects (Arthropoda: Hexapoda) of Al-Baha Province, Kingdom of Saudi Arabia (KSA) was published in 2013 and contained a total of 582 species. In the present study, 142 species belonging to 51 families and representing seven orders are added to the fauna of Al-Baha Province, bringing the total number of species now recorded from the province to 724 . The reported species are assigned to recognized regional zoogeographical regions. Seventeen of the species are recorded for the first time for KSA, namely: Platypleura arabica Myers [Cicadidae, Hemiptera]; Cletomorpha sp.; Gonocerus juniperi Herrich-Schäffer [Coreidae, Hemiptera]; Coranus lateritius (Stål); Rhynocoris bipustulatus (Fieber) [Reduviidae, Hemiptera]; Cantacader iranicus Lis; Dictyla poecilla Drake \& Hill [Tingidae, Hemiptera]; Mantispa scabricollis McLachlan [Mantispidae, Neuroptera]; Cerocoma schreberi Fabricius [Meloidae, Coleoptera]; Platypus parallelus (Fabricius) [Curculionidae, Coleoptera]; Zodion cinereum (Fabricius) [Conopidae, Diptera]; Ulidia ?ruficeps Becker [Ulidiidae, Diptera]; Atherigona reversura Villeneuve [Muscidae, Diptera]; Aplomya metallica (Wiedemann); Cylindromyia sp. [Tachinidae, Diptera]; Messor semirufus (André); and Nesomyrmex denticulatus (Mayr) [Formicidae, Hymenoptera]. Also, the reduviid bug Dasycnemus sahlbergi Bergroth, that was questionably recorded from KSA is confirmed herein as occurring in KSA. The generic name Peirates (Reduviidae, Hemiptera) and the specific name of Apis florea Fabricius (Apidae, Hymenoptera) were inadvertently misspelled in the original list and are corrected here. Zoogeographically, the present study supports the original study conclusion that the insect species composition of Al-Baha Province is strongly affiliated with the Afrotropical Region rather than to the Palearctic Region or the Eremic Zone. Our insect surveys emphasize the fact that parts of the Arabian Peninsula, including AlBaha Province, ought to be considered part of the Afrotropical Region rather than in the Palaearctic Region or the Eremic Zone.
\end{abstract}

\section{ARTICLE HISTORY}

Received 1 April 2015

Accepted 30 September 2015

Online 9 December 2015

\section{KEYWORDS}

Palaearctic; Afrotropical; Eremic; insect species; Arabian Peninsula; Tihama; Al-Sarah; Al-Sarawat Mountains 


\section{Introduction}

El-Hawagry et al. (2013) surveyed the insect fauna of Al-Baha Province, Kingdom of Saudi Arabia (KSA), reporting a total number of 582 species belonging to 129 families and representing 17 orders. Since this publication, new collections of insects were made in the province revealing additional insect records, as reported herein.

Al-Baha Province (Figure 1) is situated in the south-western part of KSA between the Holy Makkah and Asir regions (Doha 2009), It is the smallest province in KSA (about 10,362 km²), situated at $41-42^{\circ} \mathrm{E}$ and $19-20^{\circ} \mathrm{N}$. It is characterized by natural tree cover and agricultural plateaus. The province is divided by huge and steep rocky mountains into two main sectors, a lowland coastal plain in the west, known as 'Tihama', and a mountainous area with an elevation of 1500-2450 $\mathrm{m}$ above sea level at the east, known as 'Al-Sarat' or 'Al-Sarah' which form a part of Al-Sarawat Mountains range (Ibrahim and Abdoon 2005; Alahmed et al. 2010;

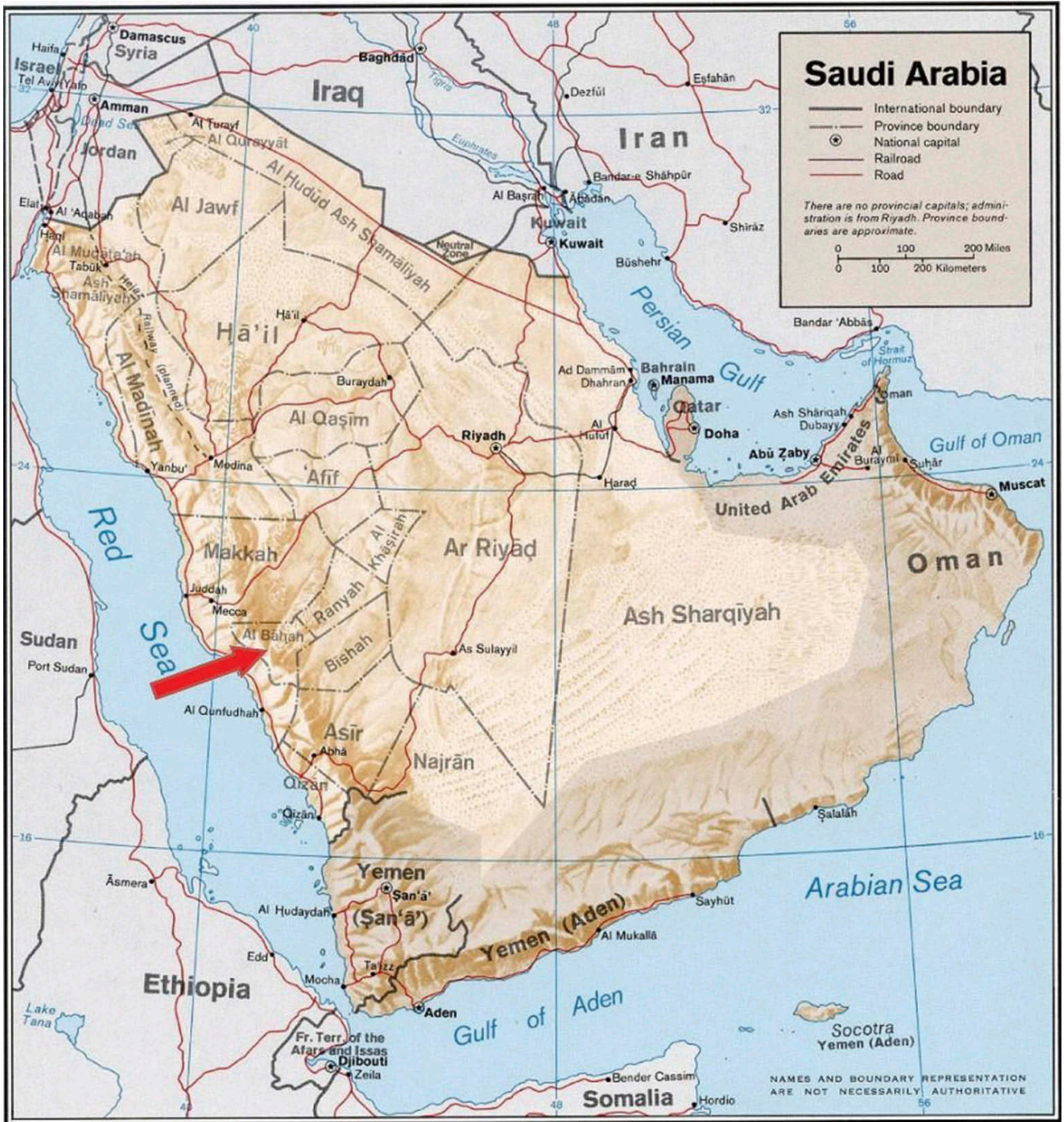

Figure 1. Map of Kingdom of Saudi Arabia showing Al-Baha Province. 
El-Hawagry et al. 2013). For more details on the topography and climate of Al-Baha Province, see our previous study (El-Hawagry et al. 2013).

Al-Baha Province (Figure 2) consists of six main districts, four of which are located in Al-Sarah sector beside downtown 'Al-Baha', i.e. Al-Aqiq, Al-Mandaq, Al-Qura, and Baljurashi, while two of the districts are located in Tihama sector, namely Al-Mekhwa including Dhee Ain Village (The Marble Village), and Qelwa (El-Hawagry et al. 2013).

Many authors assume that the Afrotropical Region covers all of Africa south of the Sahara, with the island of Madagascar and the nearby smaller islands, and include parts of the Arabian Peninsula in the region, but there seems to be no agreement as to how much. This may be deduced from the fact that the south-western and southern parts of the Arabian Peninsula including the study area are strongly influenced by a subtropical to tropical climate with spring and summer rains, and are thus dominated by a xeromesic tropical flora of palaeotropical origin, that in fact represents the impoverished northern part of an African flora. Examples of species with this conspicuous distribution pattern, linking south-west Arabia with the other side of the Red Sea, are Acacia spp., Aloe spp., Celosia spp., Celtis spp., Commiphora spp., succulent Euphorbia spp., Grewia spp., Kalanchoe spp., Nuxia spp., Olea spp., Sansevieria spp., and semi-evergreen sclerophyllous woodlands of the montane vegetation. Nevertheless, Mediterranean and northern temperate floristic elements are well represented in the study area with many plant species including Lavandula spp., Nepeta sheilae, Erica arborea, Scabiosa,

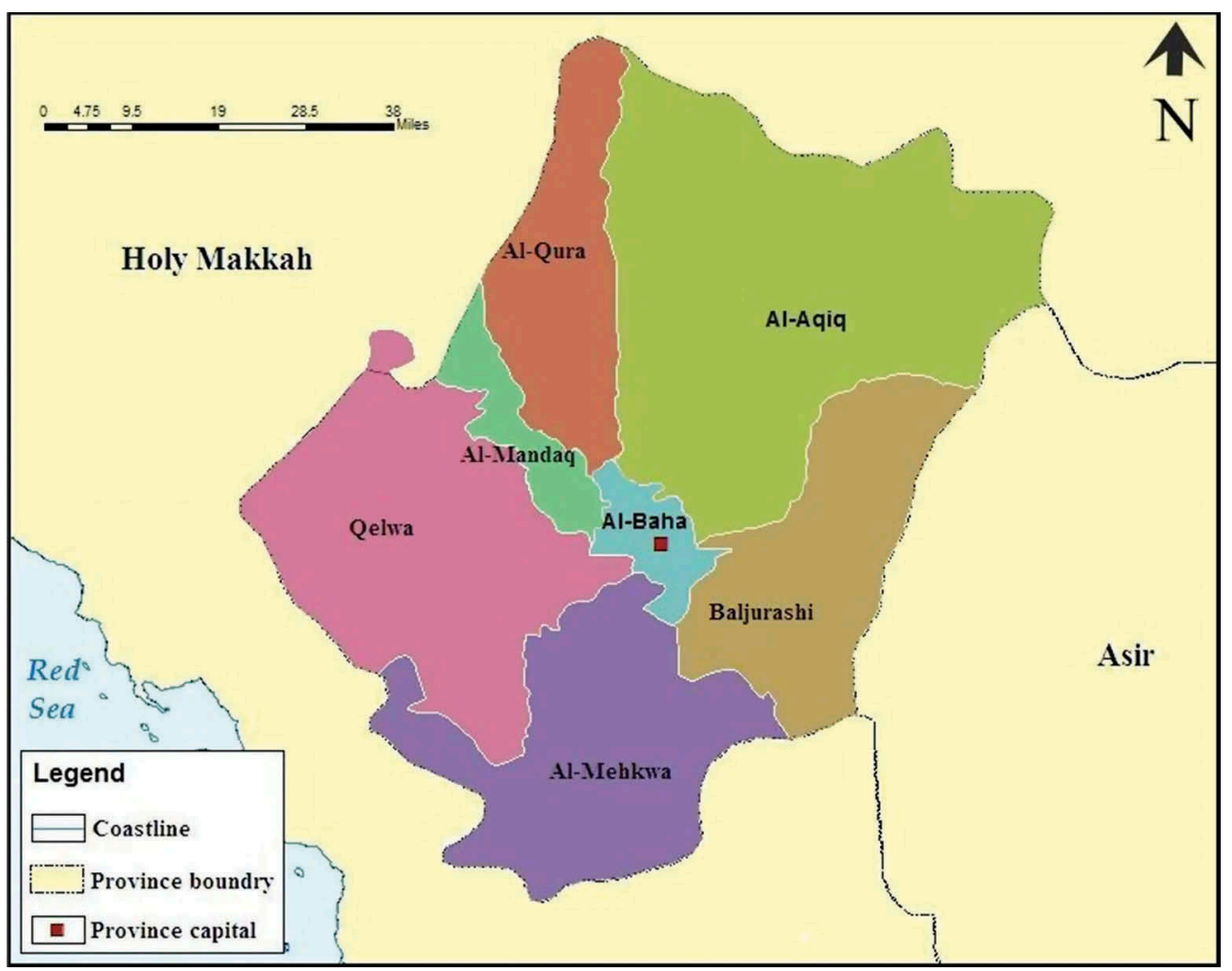

Figure 2. Map of Al-Baha Province showing its main districts. 
Silene, Trifolium, Arabis alpine, Peucedanum and Pimpinella (Ghazanfar and Fisher 1998). Sclater (1858) and Wallace (1876) proposed the classical zoogeographical regions and placed the northern border of the Afrotropics along the Tropic of Cancer. Accordingly, Al-Baha Province was included in the Afrotropical Region, and the northern limit of the Afrotropical Region was placed in Taif area, about $200 \mathrm{~km}$ north to Al-Baha (Hölzel 1998). Crosskey (1980) used the northern boundaries of Yemen as the regional boundary between the Afrotropical and Palaearctic parts in the Arabian Peninsula. Extensive sampling of Diptera in the Arabian Peninsula by many authors, especially in Yemen, Oman, the United Arab Emirates and south-western mountains of KSA, have raised some interesting questions in respect to the true extent of the Afrotropical Region in this important transitional zone, indicating that Wallace's (1876) concept of the extent of the Afrotropical Arabian Peninsula is more accurate than Crosskey's (1980) limited concept of Yemen alone (Kirk-Spriggs and McGregor 2009). However, Uvarov (1938), Greathead (1980) and Larsen (1984) agreed that the south-western part of KSA including Al-Baha should be united with the central Arabian deserts which are either considered as a part of the Palaearctic or by some authors as an autonomous Eremic Zone (also called the Saharo-Sindian faunal region). The study of El-Hawagry et al. (2013) confirmed that the insect species composition in Al-Baha Province is representative of species typically considered Afrotropical elements, as is the present list.

\section{Material and methods}

Insect material for the present study was collected extensively from different localities in Al-Baha Province from 2011 to 2014 by the authors using sweep and aerial nets, bait traps, beating sheets, soil examination, hand picking, light traps, Malaise traps, pitfall traps, sticky traps, tray sifting of debris, and yellow pan traps (Evans 1975; Borror et al. 1989). For more details on the collecting methods for each insect group, see our previous study (ElHawagry et al. 2013). Some of the specimens collected and deposited in King Saud University Museum of Arthropods were inadvertently not treated in our previous study (El-Hawagry et al. 2013). Literature records of some species are also noted.

All taxa are arranged in alphabetical order. Sites and dates of collection are included for the purpose of mapping distribution and activity periods of species in the study area. Each collection site is followed, between parentheses, by the district to which it belongs in Al-Baha Province. Also, the coordinates of each site are given. Data from 'Jebel Shada Al A'la', a protected area situated at the south-western Al-Baha Province, has been excluded from this study and will be published later.

Zoogeographical affiliations of species reported in each of the two main sectors of the province were detected using world catalogues and counted to calculate the percentage of Afrotropical, Palaearctic or Oriental elements in each sector. These percentages give a picture about the extent of zoogeographical affinity in each sector.

Images of newly recorded species were made using a Leica MZ 125 stereo-binocular microscope (Leica Microsystems Ltd, St. Gallen, Switzerland) fitted with a digital camera (Q-imaging Micro Publisher 5.0 RTV; Zerene Systems LLC, Richland, WA, USA) at the Plant Protection Department, College of Food and Agriculture Sciences, King Saud University. Photo automontage was performed by Zerene stacker program version 1.04 (http:// zerenesystems.com/cms/stacker). 
Many earlier studies were consulted in order to identify species collected or to determine species previously recorded from Al-Baha. These studies include Alahmed et al. (2010), Collingwood (1985), Collingwood and Agosti (1996), Cranston and Judd (1989), Dawah (2011), Dawah and Abdullah (2006), Dawah and Abdullah (2008), Deeming and Al-Dhafer (2012), El-Hawagry and Al Dhafer (2015), Greathead (1980, 1988), Hölzel (1983, 1998), Larsen (1984), Leclercq, Lelej and van Harten (2006), Lis (1999), Mei and Stuke (2008), Merz and Dawah (2005), Morgulis and Freidberg (2014), Moulet et al. (2014), Pape (1996), Setyaningrum and Al Dhafer (2014), Sharaf, Aldhafer, et al. (2014), and Steyskal (1968), Uvarov (1952).

All insect materials are deposited in King Saud University Museum of Arthropods, Riyadh, Saudi Arabia (KSMA).

\title{
Results
}

A total number of 142 insect species (four identified to the subspecies level and 18 identified only to the genus level) belonging to 51 families and representing seven orders are added to the fauna of Al-Baha Province by the present study.

Most species reported here are typical taxa of the Afrotropical Region rather than those associated with the Palearctic Region or the Eremic Zone. This affinity was slightly greater in Tihama (62\%) than in Al-Sarah (58\%). The study showed Palaearctic elements comprising $25 \%$ or less in both areas. There were a few Oriental elements (6\% or less) (Table 1$)$.

All additions and corrections are presented in the following text.

\author{
Class INSECTA \\ Subclass PTERYGOTA \\ Division EXOPTERYGOTA \\ Order ORTHOPTERA \\ Suborder CAELIFERA \\ Family ACRIDIDAE \\ Subfamily ACRIDINAE \\ Tribe Phlaeobini
}

Duroniella acuta Uvarov, 1952

Qelwa City (Qelwa) $19.949727^{\circ} \mathrm{N}, 41.240745^{\circ}$ E: May 2013

Table 1. Zoogeographic affinities of the insect species added to the fauna of Al-Baha Province by this study.

\begin{tabular}{lcc}
\hline & \multicolumn{2}{c}{ Affinities (\%) } \\
\cline { 2 - 3 } Region & Tihama sector & Al-Sarah sector \\
\hline Afrotropical & 62 & 58 \\
Palaearctic & 16 & 25 \\
Oriental & 5 & 6 \\
Undetermined & 17 & 11 \\
\hline
\end{tabular}




\section{Order HEMIPTERA \\ Suborder AUCHENORRHYNCHA \\ Family CICADIDAE \\ Subfamily CICADINAE \\ Tribe Platypleurini \\ Platypleura arabica Myers}

(Figure 3)

Al-Mekhwa-Namarah Road (Al-Mekhwa) 19.672591 N, 41.565185 E: June 2011. [First record in KSA].

Qelwa City (Qelwa) $19.949727^{\circ} \mathrm{N}, 41.240745^{\circ} \mathrm{E}$, Adhom Road (Qelwa) 19.992703

N, 41.182981 E: November 2012.

\section{Family CALISCELIDAE \\ Subfamily OMMATIDIOTINAE \\ Tribe Adenissini \\ Adenissus riadicus Dlabola}

Al-Baha (Downtown) 20.018129 N, 41.470771 E: July 2012.

\section{Suborder HETEROPTERA \\ Family BERYTIDAE \\ Subfamily METACANTHINAE \\ Metacanthus mollis Stusák}

Dhee Ain (Al-Mekhwa) 19.55459 N, 41.26302 E: May 2011.

$$
\begin{gathered}
\text { Family COREIDAE } \\
\text { Subfamily PSEUDOPHLOEINAE } \\
\text { Tribe Clavigrallini } \\
\text { Clavigralla griseola (Linnavouri) }
\end{gathered}
$$

Dhee Ain (Al-Mekhwa) 19.55459 N, 41.26302 E: May 2011.

\section{Subfamily COREINAE \\ Tribe Gonocerini \\ Cletomorpha sp.}

(Figure 4)

Dhee Ain (Al-Mekhwa) $19.55459^{\circ} \mathrm{N}, 41.26302^{\circ} \mathrm{E}$ : March-May 2011. [First record in KSA].

\section{Cletus binotulatus obtusulus Linnavuori}

Amadan (Al-Mandaq) $20.144656^{\circ} \mathrm{N}, 41.281698^{\circ} \mathrm{E}$, Baljurashi City (Baljurashi) $19.877804^{\circ}$

N, 41.571622 ${ }^{\circ}$, Ghabet Raghdan (Al-Baha City) $20.00575^{\circ} \mathrm{N}, 41.26974^{\circ}$ E: May 2010. Dhee Ain (Al-Mekhwa) 19.55459 ${ }^{\circ}$ N 41.26302 ${ }^{\circ}$ E: January-October 2012. 

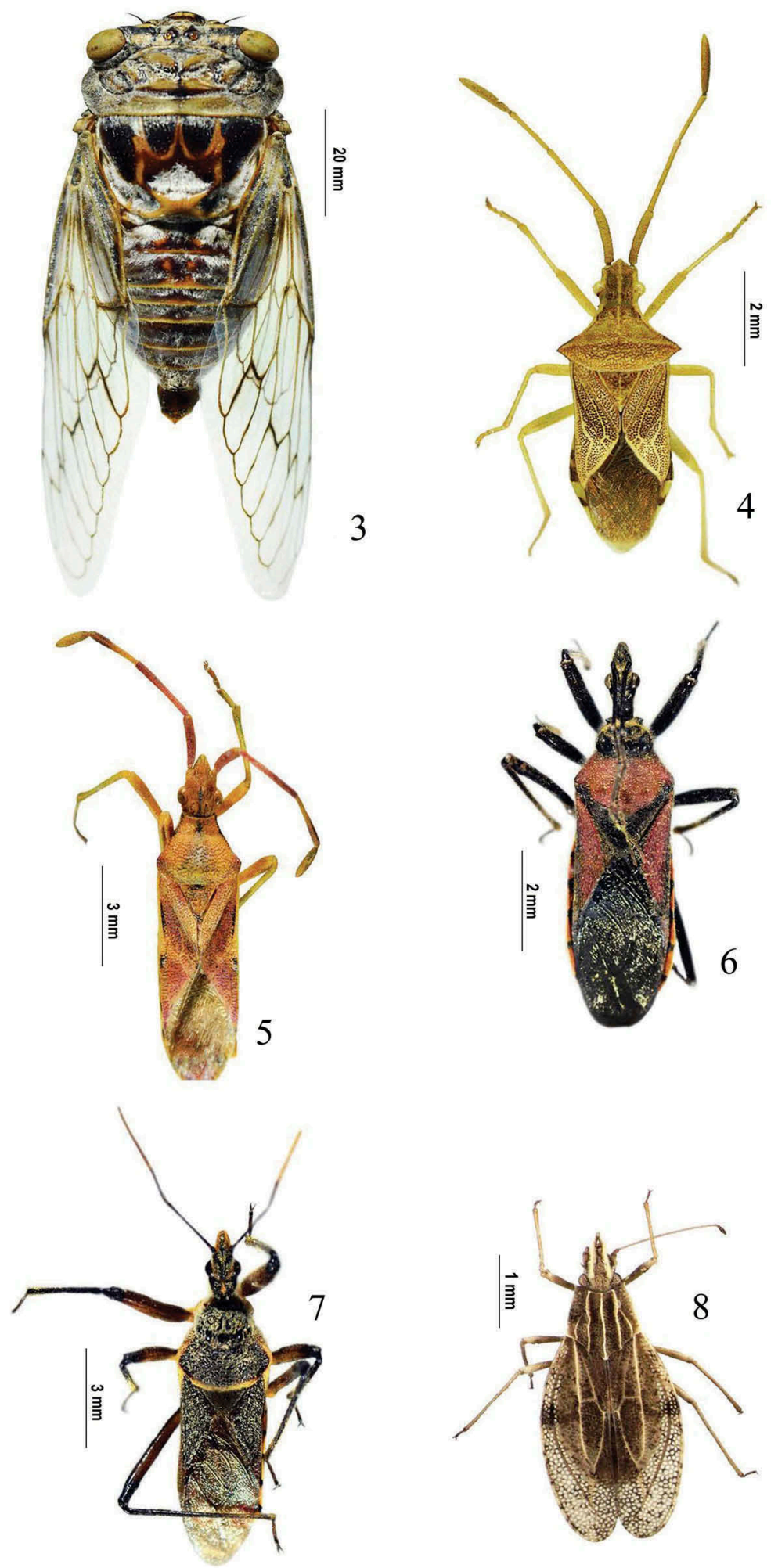

Figures 3-8. 3, Platypleura arabica Myers; 4, Cletomorpha sp.; 5, Gonocerus juniperi Herrich-Schäffer; 6, Coranus lateritius (Stål); 7, Rhynocoris bipustulatus (Fieber); 8, Cantacader iranicus Lis. 


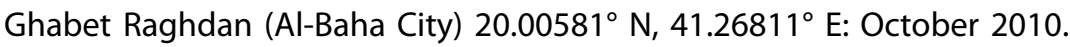

\title{
Gonocerus juniperi Herrich-Schäffer
}

(Figure 5)

Ghabet Raghdan (Al-Baha City) 20.00575 N, 41.26974 E: October 2010.

[First record in KSA].

$$
\begin{gathered}
\text { Family CYDNIDAE } \\
\text { Subfamily CYDNINAE } \\
\text { Tribe Geotomini } \\
\text { Aethus hispidulus (Klug) }
\end{gathered}
$$

Al-Mekhwa City (Al-Mekhwa) 19.770576 N, 41.434293 E: April 2011.

\section{Aethus ostiolatus Signoret}

Al-Mekhwa City (Al-Mekhwa) 19.770576 N, 41.434293 E: April 2012.

Dhee Ain (Al-Mekhwa) 19.55459 ${ }^{\circ}$ N, 41.26302 ${ }^{\circ}$ E: May 2012.

\section{Alonips intrusus (Wagner)}

Al-Mekhwa City (Al-Mekhwa) 19.770576 N, 41.434293 E: April 2012.

\section{Geotomus sp.}

Dhee Ain (Al-Mekhwa) 19.55459 N, 41.26302 E: May 2011.

$$
\text { Macroscytus brunneus (Fabricius) }
$$

Al-Baha (Downtown) 20.018129 N, 41.470771 E: June 2012.

\author{
Family HEBRIDAE \\ Subfamily HEBRINAE \\ Hebrus pusillus arabicus Linnavouri
}

Wadi Turabet Zahran (Al-Mandaq) 20.12397 ${ }^{\circ}$ N 41.17176 E: March 2012. Madhas Dam (Al-Mandaq) 20.222058 ${ }^{\circ}$ N 41.274117 E: March 2012.

\section{Family MESOVELIIDAE \\ Mesovelia vittigera Horvath}

Wadi Turabet Zahran (Al-Mandaq) $20.12397^{\circ}$ N, 41.17176 E: October 2010. 


\title{
Subfamily LACCCORINAE
}

Heleocoris minusculus (Walker)

Dhee Ain (Al-Mekhwa) 19.55459 N, 41.26302 E: March 2012.

\section{Family NEPIDAE \\ Subfamily NEPINAE \\ Tribe Nepini \\ Laccotrephes fabricii Stål}

Wadi Turabet Zahran (Al-Mandaq) 20.12397 N, 41.17176 E: October 2010.

\author{
Family NOTONECTIDAE \\ Subfamily NOTONECTINAE \\ Tribe Notonectini \\ Enithares sobria (Stål)
}

Wadi Turabet Zahran (Al-Mandaq) 20.12397 ${ }^{\circ}$, 41.17176 E: October 2010.

Notonecta arabiensis Hungerford

Wadi Turabet Zahran (Al-Mandaq) 20.12397 N, 41.17176 E: May 2011.

\author{
Family REDUVIIDAE \\ Subfamily HARPACTORINAE \\ Tribe Harpactorini \\ Coranus angulatus Stål
}

Al-Baha (Downtown) 20.018129 N, 41.470771 E: May 2011.

Wadi Turabet Zahran (Al-Mandaq) 20.12397 N, 41.17176 E: May 2011.

Coranus kiritshenkoi Bergevin

Wadi Jalla: May 2011

\section{Coranus lateritius (Stål)}

(Figure 6)

Wadi Turabet Zahran (Al-Mandaq) $20.12397^{\circ} \mathrm{N}, 41.17176^{\circ} \mathrm{E}$ : May 2011. [First record in KSA] Rhynocoris bipustulatus (Fieber)

(Figure 7)

Dhee Ain (Al-Mekhwa) $19.55459^{\circ} \mathrm{N}, 41.26302^{\circ} \mathrm{E}$ : May 2011. [First record in KSA]

Cosmolestes pictus (Klug)

Wadi Turabet Zahran (Al-Mandaq) 20.12397 N, 41.17176 E: May 2011. 

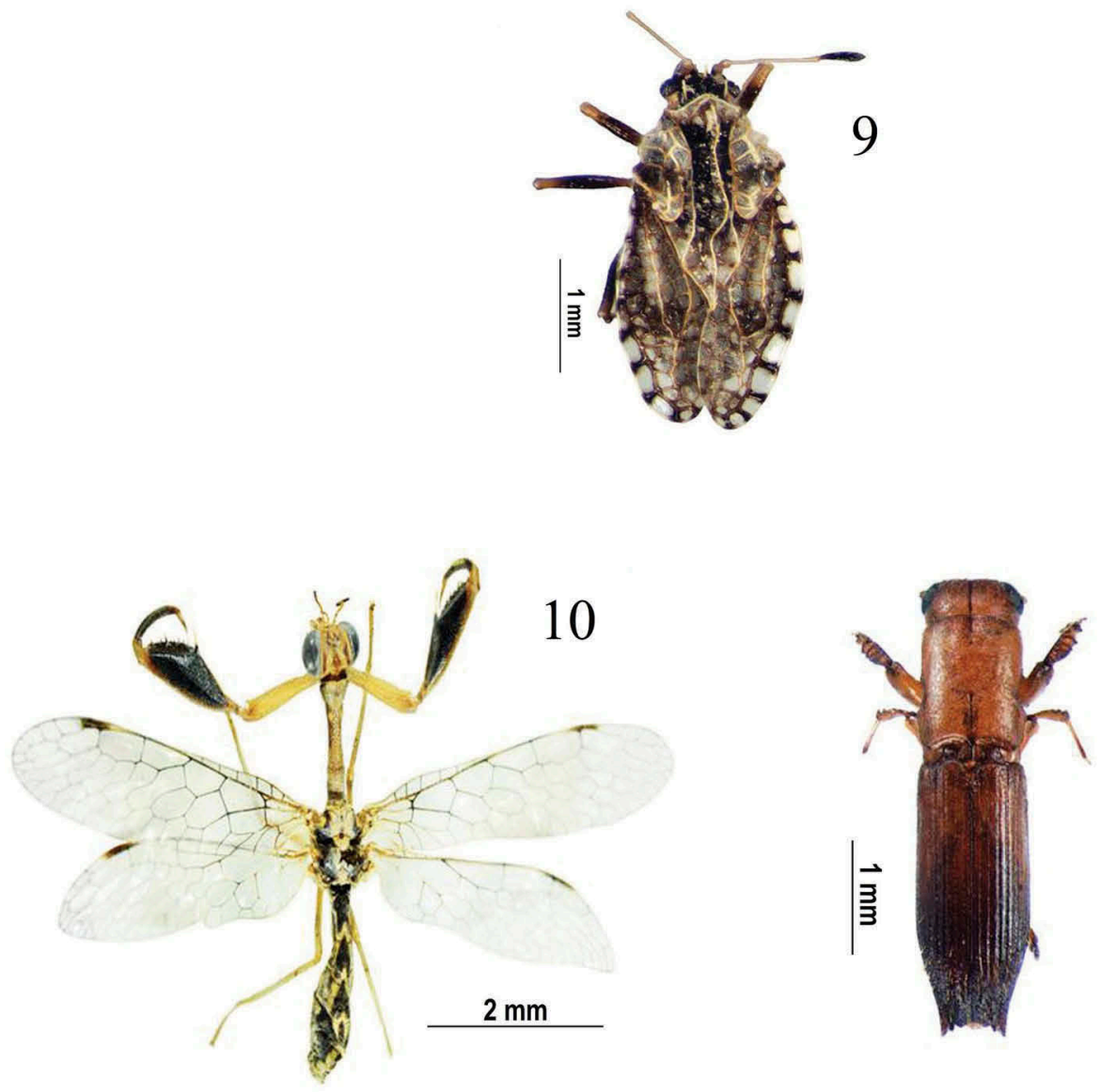

11
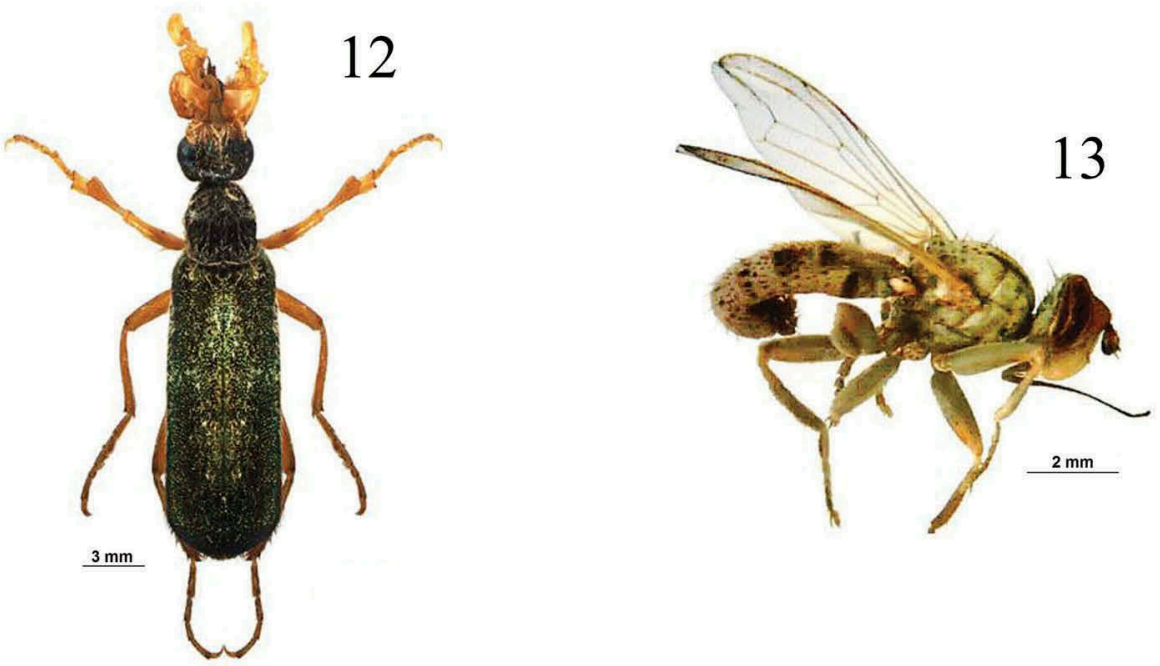

Figures 9-13. 9, Dictyla poecilla Drake \& Hill; 10, Mantispa scabricollis McLachlan; 11, Platypus parallelus (Fabricius); 12, Cerocoma schreberi Fabricius; 13, Zodion cinereum (Fabricius). 


\title{
Tribe Dasycnemini \\ Dasycnemus sahlbergi Bergroth
}

Dhee Ain (Al-Mekhwa) 19.55459 N, 41.26302 E: September 2011.

Subfamily PEIRATINAE

Peirates strepitans Rambur

[Inadvertently reported as Pirates in El-Hawagry et al. (2013).

Wadi Genouna $19.454706^{\circ}$ N, 41.603165 E: May 2012.

Family RHYPAROCHROMIDAE

Subfamily RHYPAROCHROMINAE

Tribe Lethaeini

Lethaeus fulvovarius Puton

Ghabet Raghdan (Al-Baha City) 20.00575 N, 41.26974 E: May 2011.

\author{
Family TINGIDAE \\ Subfamily CANTACADERINAE \\ Tribe Cantacaderini \\ Cantacader iranicus Lis
}

(Figure 8)

Dhee Ain (Al-Mekhwa) $19.55459^{\circ} \mathrm{N}, 41.26302^{\circ} \mathrm{E}$ : March 2012. [First record in KSA].

Subfamily TINGINAE

Tribe Tingini

Compseuta montandoni Distant

Dhee Ain (Al-Mekhwa) 19.55459 N, 41.26302 ${ }^{\circ}$ E: May-October 2010.

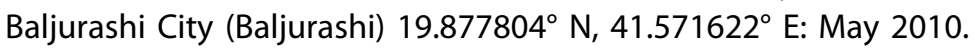

Phaenotropis cleopatra (Horváth)

Ghabet Raghdan (Al-Baha City) 20.00575 N, 41.26974 E: May 2010.

\section{Plerochila australis (Distant)}

Wadi Al-Zarayeb (Al-Mandaq) 20.03601 N, 41.23193 E: May 2014.

Tribe Tingitini

Dictyla nassata (Puton)

Al-Atawla (Al-Qura) 20.523609 N, 41.5954626 E: April 2013.

Dhee Ain (Al-Mekhwa) 19.55459 $\mathrm{N}, 41.26302^{\circ} \mathrm{E}$ : March 2012.

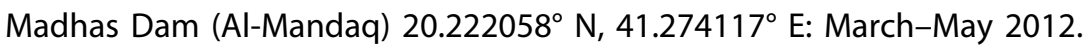


Dictyla poecilla Drake \& Hill

(Figure 9)

Dhee Ain (Al-Mekhwa) $19.55459^{\circ} \mathrm{N}, 41.26302^{\circ} \mathrm{E}$ : March 2012. [First record in KSA].

Wadi Turabet Zahran (Al-Mandaq) 20.12397 ${ }^{\circ}$ N 41.17176 E: March 2013.

\title{
Suborder STERNORRHYNCHA \\ Family PSYLLIDAE \\ Diaphorina citri Kuwayama
}

Al-Mekhwa City (Al-Mekhwa) 19.770576 N, 41.434293ํㅡ: September 2012.

\section{Division: ENDOPTERYGOTA \\ Order NEUROPTERA \\ Family ASCALAPHIDAE \\ Subfamily ASCALAPHINAE \\ Ascalaphus festivus (Rambur)}

Dhee Ain (Al-Mekhwa) 19.55459 $\mathrm{N}, 41.26302^{\circ} \mathrm{E}$ : March 2012.

Wadi Turabet Zahran (Al-Mandaq) 20.12397 N, 41.17176 E: June 2012.

Ascalaphus krueperi (van der Weele)

Wadi Turabet Zahran (Al-Mandaq) 20.12397 N, 41.17176 E: June 2012.

Subfamily HAPLOGLENIINAE

Tmesibasis larseni Hölzel, 1983

Wadi Turabet Zahran (Al-Mandaq) 20.12397 N, 41.17176 E: June 2012.

\author{
Family MANTISPIDAE \\ Subfamily MANTISPINAE \\ Mantispa scabricollis McLachlan
}

(Figure 10)

Dhee Ain (Al-Mekhwa) $19.55459^{\circ} \mathrm{N}, 41.26302^{\circ} \mathrm{E}$ : March-May 2012. [First record in KSA]. Ghabet Raghdan (Al-Baha City) 20.00575 N, 41.26974 E: May 2011.

\author{
Order COLEOPTERA \\ Suborder POLYPHAGA \\ Family CURCULIONIDAE \\ Subfamily PLATYPODINAE \\ Tribe Platypodini \\ Platypus parallelus (Fabricius)
}

(Figure 11)

Dhee Ain (Al-Mekhwa) $19.55459^{\circ} \mathrm{N}, 41.26302^{\circ} \mathrm{E}$ : March-May 2011. [First record in KSA]. 


\title{
Family MELOIDAE \\ Subfamily MELOINAE \\ Tribe Cerocomini \\ Cerocoma schreberi Fabricius
}

(Figure 12)

Ghabet Shahba (Al-Baha City) $20.02723^{\circ} \mathrm{N}, 41.28565^{\circ} \mathrm{E}$ : May-June 2012. [First record in KSA].

Family SCARABAEIDAE

Subfamily CETONIINAE

Pachnoda fasciata Fabricius

Aqabet Al-Baha-Tihama $20.00000^{\circ} \mathrm{N}, 41.43758^{\circ} \mathrm{E}$ : March 2012.

\author{
Order LEPIDOPTERA \\ Suborder RHOPALOCERA \\ Family LYCAENIDAE \\ Subfamily THECLINAE \\ Tribe THECLINI \\ Deudorix livia (Klug)
}

Aqabet Al-Baha-Tihama 20.00000 N, 41.43758E: March 2012.

\section{Hypolycaena pachalica Butler}

Aqabet Al-Baha-Tihama $20.00000^{\circ} \mathrm{N}, 41.43758^{\circ} \mathrm{E}$ : March 2012.

Iolaus nursei Butler

Aqabet Al-Baha-Tihama $20.00000^{\circ} \mathrm{N}, 41.43758^{\circ} \mathrm{E}$ : March 2012.

$$
\begin{gathered}
\text { Family NYMPHALIDAE } \\
\text { Subfamily NYMPHALINAE } \\
\text { Tribe Junoniini } \\
\text { Junonia orithya here Lang }
\end{gathered}
$$

Aqabet Al-Baha-Tihama 20.00000 N, 41.43758E: March 2012.

\section{Precis limnoria (Klug)}

Aqabet Al-Baha-Tihama 20.00000 N, 41.43758 E: March 2012.

Subfamily SATYRINAE

$$
\text { Ypthima asterope (Klug) }
$$

Aqabet Al-Baha-Tihama 20.00000 N, 41.43758E: March 2012. 


\title{
Suborder HETEROCERA \\ Family SPHINGIDAE \\ Subfamily SMERINTHINAE \\ Tribe Smerinthini \\ Pseudoclanis molitor subviridis Talbot
}

Dhee Ain (Al-Mekhwa) 19.55459 ${ }^{\circ}$ N, 41.26302 ${ }^{\circ}$ E: March 2010.

Family ZYGAENIDAE

Subfamily ZYGAENINAE

Reissita simonyi yemenicola (Tremewan)

Ghabet Raghdan (Al-Baha City) 20.00575 N, 41.26974 E: May 2010.

Ghabet Shahba (Al-Baha City) $20.02723^{\circ} \mathrm{N}, 41.28565^{\circ}$ E: June 2010.

\author{
Order DIPTERA \\ Suborder NEMATOCERA \\ Family BIBIONIDAE \\ Dilophus tridentatus Walker
}

Wadi Turabet Zahran (Al-Mandaq) 20.12397 N, 41.17176 E: March 2013.

Dilophus sp.

Al-Dhafeer (Al-Baha City) 20.010225 N, 41.468368 E: May 2013.

Family CERATOPOGONIDAE

Culicoides (Avaritia) imicola Kieffer

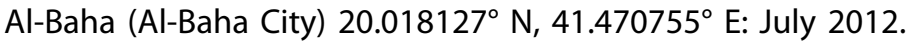

Family CHIRONOMIDAE

Subfamily CHIRONOMINAE

Tribe Chironomini

Chironomus calipterus Kieffer

Al-Mekhwa City (Al-Mekhwa) 19.770576 N, 41.434293 E: November 1980.

Chironomus callichirus Kieffer

Al-Baha (Al-Baha City) 20.018332 N, 41.470578 E: August 1984.

Polypedilum (Pentapedilum) alticola Kieffer

Al-Baha (Al-Baha City) 20.018332 N, 41.470578 E: July 1981.

Subfamily TANYPODINAE

Larsia rutshuruiensis (Goetghebuer) 
Al-Mandaq (Al-Mandaq) 20.113122 N, 41.2854791 E: April 1980.

\title{
Subfamily ORTHOCLADIINAE
}

Paratrichocladius micans (Kieffer)

Dhee Ain (Al-Mekhwa) $19.55459^{\circ} \mathrm{N}, 41.26302^{\circ} \mathrm{E}$ : August ?.

\section{Suborder BRACHYCERA \\ Family ASILIDAE \\ Subfamily APOCLEINAE \\ Promachus sp.}

Al-Mekhwa City (Al-Mekhwa) 19.770576 N, 41.434293 E: February-March 2012.

\author{
Family BOMBYLIIDAE \\ Subfamily BOMBYLIINAE \\ Tribe Bombyliini \\ Bombylella delicata (Wiedemann)
}

Ghabet Shahba (Al-Baha City) 20.02723 N, 41.28565 E: May-June 2012.

Bombylius discoidea Fabricius

Ghabet Shahba (Al-Baha City) 20.02723 N, 41.28565 E: May-June 2012.

\section{Subfamily ANTHRACINAE \\ Tribe Exoprosopini \\ Exoprosopa linearis Bezzi}

Al-Mekhwa City (Al-Mekhwa) 19.770576 N, 41.434293 E: March 2012.

Exoprosopa minos (Meigen)

Aqabet Al-Baha-Tihama 20.00000 N, 41.43758E: April-May 2012.

Family DOLICHOPODIDAE

Subfamily DIAPHORINAE

Asyndetus albifacies Parent

Al-Mekhwa City (Al-Mekhwa) 19.770576 N, 41.434293 E: April 2012.

\section{Family HYBOTIDAE \\ Subfamily TACHYDROMIINAE}

Platypalpus sp.

Al-Mekhwa City (Al-Mekhwa) 19.770576 N, 41.434293 E: April-May 2012. 


\title{
Family TABANIDAE \\ Subfamily EXORISTINAE \\ Gonia bimaculata Wiedemann
}

Ghabet Shahba (Al-Baha City) 20.02723 N, 41.28565 E: April 2012.

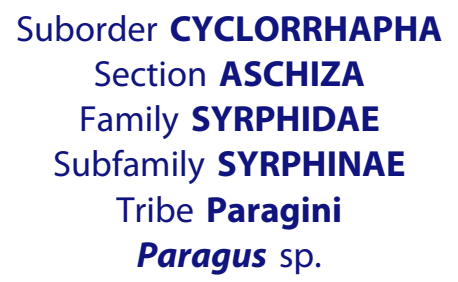

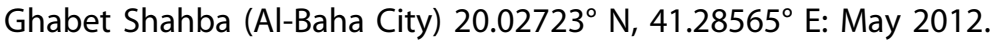

Tribe Syrphini

Asarkina sp.

Ghabet Shahba (Al-Baha City) $20.02723^{\circ}$ N, 41.28565 E: February 2012.

Section SCHIZOPHORA
Subsection ACALYPTRATAE
Family CARNIDAE
Carnus hemapterus Nitzsch

Al-Mekhwa City (Al-Mekhwa) 19.770576º N, 41.434293 E: April-May 2013.

Meoneura nitidiuscula Collin

Al-Mekhwa City (Al-Mekhwa) 19.770576 N, 41.434293 E: April-May 2013.

\author{
Family CHLOROPIDAE \\ Subfamily CHLOROPINAE \\ Metopostigma sabulona Becker
}

Wadi Turabet Zahran (Al-Mandaq) 20.12397 N, 41.17176 E: March-April 2012.

Mepachymerus baculus Speiser

Al-Dhafeer (Al-Baha City) 20.010225 N, 41.468368 E: October 2012.

Pachylophus pellucidus Becker

Al-Mekhwa City (Al-Mekhwa) $19.770576^{\circ}$ N, 41.434293 E: May 2012 


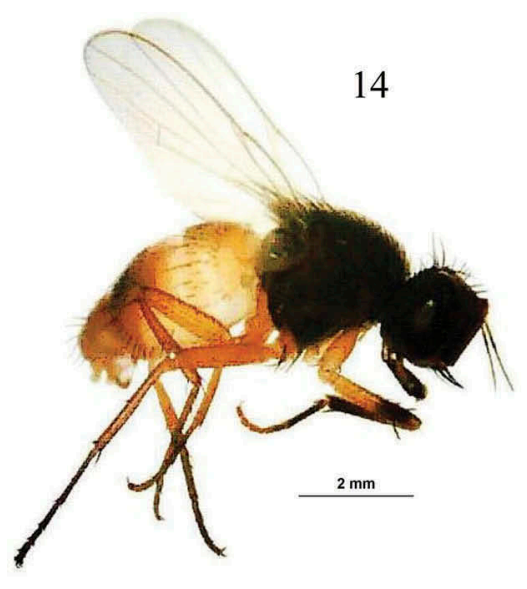

16

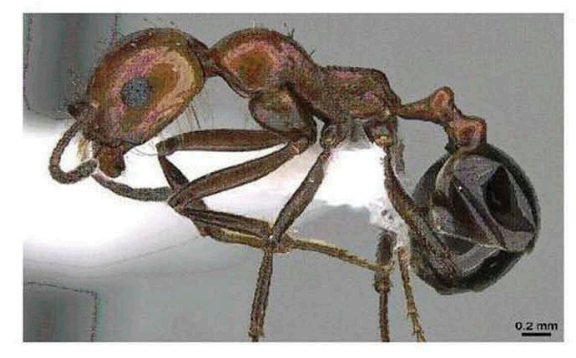

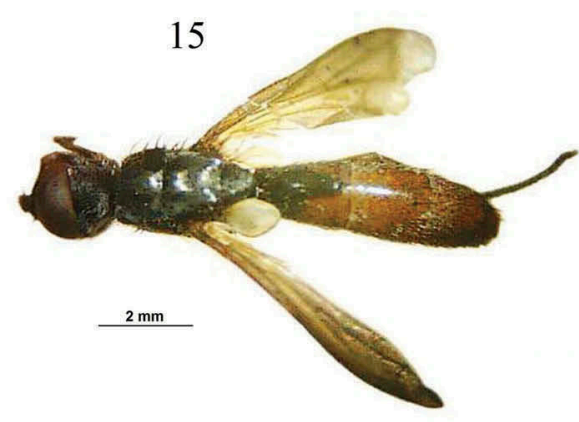

17

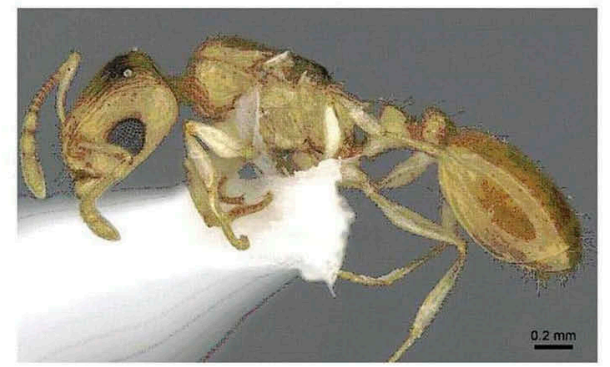

Figures 14-17. 14, Atherigona reversura Villeneuve; 15, Cylindromyia sp.; 16, Messor semirufus (André), worker [CASENT 0906385, Photographer: Estella Ortega, copyright: www.antweb.org]; 17, Nesomyrmex denticulatus (Mayr), dealate queen [CASENT 0906381, Photographer: Estella Ortega, copyright: www.antweb.org].

\section{Pachylophus proximus Adams}

Wadi Turabet Zahran (Al-Mandaq) 20.12397 N, 41.17176 E: March-April 2012.

\section{Subfamily OSCINELLINAE \\ Anatrichus pygmaeus Lamb}

Al-Mekhwa City (Al-Mekhwa) 19.770576 N, 41.434293 E: April 2012.

\section{Scoliophthalmus micantipennis Duda}

Al-Mekhwa City (Al-Mekhwa) 19.770576 N, 41.434293 E: April 2012.

\section{Scoliophthalmus trapezoides Becker}

Al-Mekhwa City (Al-Mekhwa) 19.770576 N, 41.434293 E: April 2012. 


\title{
Subfamily MYOPINAE \\ Tribe Zodionini \\ Zodion cinereum (Fabricius)
}

(Figure 13)

Ghabet Shahba (Al-Baha City) 20.02723 N, 41.28565 E: May-June 2012.

[First record in $\mathrm{KSA}$ ].

\section{Family DIOPSIDAE}

Diopsis collaris Westwood

Al-Mekhwa City (Al-Mekhwa) 19.770576 N, 41.434293 E: February-March 2012. Dhee Ain (Al-Mekhwa) 19.55459 ${ }^{\circ}$ N, 41.26302 ${ }^{\circ}$ E: February-March 2012.

\section{Family EPHYDRIDAE \\ Subfamily DISCOMYZINAE \\ Psilopa nilotica (Becker)}

Al-Mekhwa City (Al-Mekhwa) 19.770576 N, 41.434293 E: February-March 2013.

\section{Subfamily HYDRELLIINAE \\ Notiphila ignobilis Loew}

Al-Mekhwa City (Al-Mekhwa) 19.770576 N, 41.434293 E: April 2012.

\section{Family LONCHAEIDAE \\ Subfamily LONCHAEINAE \\ Tribe Lonchaeini \\ Silba virescens Macquart}

Al-Mekhwa City (Al-Mekhwa) 19.770576 N, 41.434293 E: February-March 2013.

\author{
Family MILICHIIDAE \\ Subfamily MADIZINAE \\ Leptometopa rufifrons Becker
}

Aqabet Al-Baha-Tihama 20.00000 N, 41.43758 E: April-May 2013.

Subfamily MILICHIINAE

Enigmilichia dimorphica Deeming

Al-Mekhwa City (Al-Mekhwa) 19.770576 N, 41.434293 E: April 2012.

Family TEPHRITIDAE

Subfamily DACINAE

Tribe Dacini

Bactrocera cucurbitae (Coquillett) 
Al-Mekhwa City (Al-Mekhwa) 19.770576 N, 41.434293 E: February-April 2012.

\title{
Subfamily TEPHRITINAE
}

Dioxyna sp.

Ghabet Shahba (Al-Baha City) 20.02723 N, 41.28565 E: April-June 2012.

\section{Family ULIDIIDAE}

Subfamily ULIDIINAE

Tribe Ulidiini

Physiphora alceae (Preyssler)

Al-Mekhwa City (Al-Mekhwa) 19.770576 N, 41.434293 E: February-May 2013.

Ulidia ?ruficeps Becker

Al-Mekhwa City (Al-Mekhwa) $19.770576^{\circ}$ N, $41.434293^{\circ}$ E: April-May 2013. [First record in KSA]

\author{
Subsection CALYPTRATAE \\ Family ANTHOMYIIDAE \\ Subfamily ANTHOMYIINAE \\ Tribe Hydrophoriini \\ Delia sp.
}

Wadi Turabet Zahran (Al-Mandaq) 20.12397 N, 41.17176 E: March-April 2013.

\author{
Family CALLIPHORIDAE \\ Subfamily CALLIPHORINAE \\ Cordylobia anthropophaga (Blanchard)
}

Dhee Ain (Al-Mekhwa) 19.55459 N, 41.26302 E: May 2012.

Pericallimyia greatheadi Zumpt

Al-Baha (Downtown) 20.018129 N, 41.470771 E: August 2012.

\section{Subfamily CHRYSOMYINAE \\ Chrysomya bezziana Villeneuve}

Ghabet Shahba (Al-Baha City) 20.02723 N, 41.28565 E: April-May 2013.

\section{Chrysomya marginalis (Wiedemann)}

Dhee Ain (Al-Mekhwa) 19.55459 N, 41.26302 E: May 2012.

Wadi Jalla: May.

Wadi Turabet Zahran (Al-Mandaq) 20.12397 N, 41.17176 E: May 2013. 


\section{Subfamily LUCILIINAE \\ Lucilia cuprina (Wiedemann)}

Al-Mandaq (Al-Mandaq) 20.113607 N, 41.2854624 E: May-July 2012.

Dhee Ain (Al-Mekhwa) 19.55459 $\mathrm{N}, 41.26302^{\circ} \mathrm{E}$ : October 2011.

Ghabet Raghdan (Al-Baha City) $20.00575^{\circ} \mathrm{N}, 41.26974^{\circ} \mathrm{E}$ : May-June 2012. Wadi Jalla: May 2011.

Wadi Turabet Zahran (Al-Mandaq) 20.12397 N, 41.17176 E: May 2012.

\section{Subfamily POLLENIINAE \\ Pollenia dasypoda Portschinsky}

Wadi Turabet Zahran (Al-Mandaq) 20.12397 N, 41.17176 E: May 2013.

\section{Pollenia rudis (Fabricius)}

Dhee Ain (Al-Mekhwa) 19.55459 N, 41.26302 E: April-June 2012.

Wadi Jalla: May 2011.

Wadi Turabet Zahran (Al-Mandaq) 20.12397 N, 41.17176 E: May-June 2012.

\section{Subfamily RHINIINAE}

\section{Cosmina arabica Robineau-Desvoidy}

Dhee Ain (Al-Mekhwa) 19.55459 N, 41.26302 E: May-June 2012.

Wadi Jalla: May 2012.

Wadi Turabet Zahran (Al-Mandaq) 20.12397 N, 41.17176 E: May-June 2012.

$$
\text { Isomyia terminata (Wiedemann) }
$$

Wadi Turabet Zahran (Al-Mandaq) 20.12397 N, 41.17176º: May 2012.

\section{Rhinia apicalis (Wiedemann)}

Dhee Ain (Al-Mekhwa) $19.55459^{\circ} \mathrm{N}, 41.26302^{\circ} \mathrm{E}$ : May and November.

Wadi Jalla: May 2012.

\section{Stomorhina lunata (Fabricius)}

Wadi Turabet Zahran (Al-Mandaq) 20.12397 N, 41.17176 E: May 2012.

\section{Stomorhina rugosa (Bigot)}

Dhee Ain (Al-Mekhwa) 19.55459 ${ }^{\circ} \mathrm{N}, 41.26302^{\circ} \mathrm{E}$ : March-May 2012.

Wadi Turabet Zahran (Al-Mandaq) 20.12397 N, 41.17176 E: March-June 2012. 


\title{
Family MUSCIDAE \\ Subfamily ATHERIGONINAE \\ Tribe Atherigonini \\ Atherigona laevigata (Loew)
}

Wadi Turabet Zahran (Al-Mandaq) 20.12397 N, 41.17176 E: March-May 2012.

\section{Atherigona orientalis (Schiner)}

Al-Mekhwa City (Al-Mekhwa) 19.770576 N, 41.434293 E: February 2013.

Atherigona reversura Villeneuve

(Figure 14)

Al-Mekhwa City (Al-Mekhwa) $19.770576^{\circ} \mathrm{N}, 41.434293^{\circ}$ E: February 2013. [First record in $\mathrm{KSA}]$.

Subfamily MYDAEINAE

Graphomya maculata (Scopoli)

Wadi Turabet Zahran (Al-Mandaq) 20.12397 N, 41.17176 E: April 2012.

\author{
Family SARCOPHAGIDAE \\ Subfamily SARCOPHAGINAE \\ Sarcophaga destructor Malloch
}

Al-Baha (Downtown) 20.018129 N, 41.470771 E: May 2012.

\author{
Family TACHINIDAE \\ Subfamily EXORISTINAE \\ Tribe Eryciini \\ Aplomya metallica (Wiedemann) [First record in KSA]
}

Ghabet Raghdan (Al-Baha City) 20.00575 N, 41.26974 E: June 2012.

\section{Tribe Exoristini \\ Exorista xanthaspis (Wiedemann)}

Al-Mekhwa City (Al-Mekhwa) 19.770576 N, 41.434293 E: June 2012.

\section{Tribe Goniini \\ Gonia bimaculata Wiedemann}

Ghabet Shahba (Al-Baha City) 20.02723 N, 41.28565 E: May-July 2012.

\author{
Subfamily TACHININAE
}

Mintho praeceps (Scopoli)

Baljurashi City (Baljurashi) 19.877804 N, 41.571622 E: March 2013. 


\title{
Subfamily PHASIINAE \\ Tribe Cylindromyiini \\ Cylindromyia sp.
}

(Figure 15)

Al-Mekhwa City (Al-Mekhwa) $19.770576^{\circ}$ N, 41.434293 E: February-March 2012. [First record in $\mathrm{KSA}]$.

\section{Tribe Phasiini}

? Phasia sp.

Wadi Turabet Zahran (Al-Mandaq) $20.12397^{\circ} \mathrm{N}, 41.17176^{\circ}$ E: May 2012.

\section{Order HYMENOPTERA \\ Suborder APOCRITA \\ Family APIDAE \\ Subfamily APINAE \\ Tribe Apini \\ Apis florea Fabricius}

[The specific epithet was inadvertently written as florae in the original study (El-Hawagry et al. 2013)].

Al-Mekhwa City (Al-Mekhwa) 19.770576 N, 41.434293 E: February-March 2011. Wadi Jalla: May-September 2011.

Wadi Turabet Zahran (Al-Mandaq) 20.12397 N, 41.17176 E: May-October 2011.

Dhee Ain (Al-Mekhwa) 19.55459 ${ }^{\circ}$, 41.26302 ${ }^{\circ}$ : May-August 2011.

\section{Tribe Anthophorini}

Amegilla sp.

Al-Mekhwa City (Al-Mekhwa) 19.770576 N, 41.434293 E: February 2012.

\section{Tribe Eucerini}

Tetraloniella sp.

Al-Mekhwa City (Al-Mekhwa) 19.770576 N, 41.434293 E: February 2012.

\section{Tribe Melectini}

Thyreus sp.

Al-Mekhwa City (Al-Mekhwa) 19.770576 N, 41.434293 E: March 2012.

\author{
Subfamily XYLOCOPINAE \\ Tribe Ceratinini \\ Ceratina sp.
}

Al-Mekhwa City (Al-Mekhwa) 19.770576 N, 41.434293 E: February-April 2012. 


\section{Tribe Xylocopini \\ Xylocopa pubescens Spinola}

Al-Dhafeer (Al-Baha City) 20.010225 N, 41.468368 E: May 2012.

Dhee Ain (Al-Mekhwa) 19.55459 $\mathrm{N}, 41.26302^{\circ} \mathrm{E}$ : January 2011.

Wadi Turabet Zahran (Al-Mandaq) 20.12397 ${ }^{\circ}$ N 41.17176 E: March 2013.

\section{Family FORMICIDAE Subfamily DOLICHODERINAE \\ Technomyrmex setosus Collingwood, 1985}

Wadi Turabet Zahran (Al-Mandaq) 20.12397 N, 41.17176 E: March 2011.

Al-Qamh (Baljurashi) 19.807666 N, 41.705947 E: May 2010.

\section{Subfamily FORMICINAE \\ Camponotus atlantis Forel}

Al-Mandaq (Al-Mandaq) 20.113607 N, 41.2854624 E: May 2010.

\section{Camponotus ilgii Forel}

Dhee Ain (Al-Mekhwa) 19.55459 N, 41.26302 E: May 2010.

Subfamily MYRMICINAE

\section{Cardiocondyla emeryi Forel}

Al-Mandaq (Al-Mandaq) $20.113607^{\circ} \mathrm{N}, 41.2854624^{\circ} \mathrm{E}$ : May 2012, Dhee Ain

(Al-Mekhwa) $19.55459^{\circ} \mathrm{N}, 41.26302^{\circ} \mathrm{E}$ : May 2011, Wadi Turabet Zahran (Al-Mandaq) $20.12397^{\circ} \mathrm{N}, 41.17176^{\circ} \mathrm{E}:$ May 2011.

\section{Carebara arabica (Collingwood \& Van Harten)}

Dhee Ain (Al-Mekhwa) 19.55459 N, 41.26302 E: May 2011.

Messor semirufus (André)

(Figure 16)

Al-Mandaq (Al-Mandaq) $20.12163^{\circ} \mathrm{N}, 41.13906^{\circ} \mathrm{E}$ : May 2010. [First record in KSA].

Messor ebeninus Santschi

Wadi Kheira (Al-Mandaq) 20.063572 ${ }^{\circ}$ N, 41.398428 E: May 2010.

Monomorium venustum (Smith)

Dhee Ain (Al-Mekhwa) 19.55459 N, 41.26302 E: September 2011. 
Dhee Ain (Al-Mekhwa) 19.55459 N, 41.26302 E: May 2010.

\title{
Nesomyrmex denticulatus (Mayr)
}

(Figure 17)

Ghabet Shahba (Al-Baha City) $20.02723^{\circ} \mathrm{N}, 41.28565^{\circ}$ E: May 2010. [First record in KSA].

\section{Strumigenys arnoldi Forel}

Dhee Ain (Al-Mekhwa) 19.55459 N, 41.26302 E: May 2011.

Tetramorium depressiceps Menozzi

Wadi Turabet Zahran (Al-Mandaq) 20.12397 N, 41.17176 E: May 2011.

Tetramorium juba Collingwood, 1985

Wadi Jalla: April 2011.

Tetramorium caespitum (Linnaeus)

Wadi Kheira (Al-Mandaq) 20.063572 N, 41.398428 E: May 2011.

Tetramorium khyarum Bolton

Dhee Ain (Al-Mekhwa) 19.55459 N, 41.26302 E: September 2011.

\author{
Family HALICTIDAE \\ Subfamily HALICTINAE \\ Tribe Halictini \\ Lasioglossum gibber (Vachal)
}

Al-Mekhwa City (Al-Mekhwa) 19.770576 N, 41.434293 E: March 2012.

Tribe Sphecodini

Sphecodes sp.

Al-Mekhwa City (Al-Mekhwa) 19.770576 N, 41.434293 E: March 2012.

Subfamily NOMIINAE

Pseudapis sp.

Al-Mekhwa City (Al-Mekhwa) 19.770576 N, 41.434293 E: February-April 2012.

\section{Family MEGACHILIDAE Subfamily MEGACHILINAE Tribe Anthidiini \\ Anthidium waltii Spinola}

Al-Mekhwa City (Al-Mekhwa) 19.770576 N, 41.434293 E: February 2012. 


\title{
Icteranthidium ferrugineum (Fabricius)
}

Al-Mekhwa City (Al-Mekhwa) 19.770576 N, 41.434293 E: March 2012.

\section{Tribe Megachilini}

Chalicodoma sp.

Al-Mekhwa City (Al-Mekhwa) 19.770576 N, 41.434293 E: February 2012.

\author{
Family MUTILLIDAE \\ Subfamily DASYLABRINAE \\ Tribe Dasylabrini \\ Tricholabiodes niloticus Suárez
}

Baljurashi City (Baljurashi) 19.877804 N, 41.571622 E: May 2010.

Subfamily MUTILLINAE

Tribe Trogaspidiini

Trogaspidia floralis (Klug)

Baljurashi City (Baljurashi) 19.877804 N, 41.571622 E: May 2010.

Dhee Ain (Al-Mekhwa) 19.55459 ${ }^{\circ}$ N, 41.26302 ${ }^{\circ}$ E: October 2010.

\section{Subfamily MYRMILLINAE}

Omotilla conjuncta (Klug)

Baljurashi City (Baljurashi) 19.877804 N, 41.571622 E: May 2010.

Subfamily PSEUDOPHOTOPSIDINAE

Pseudophotopsis continua (Fabricius)

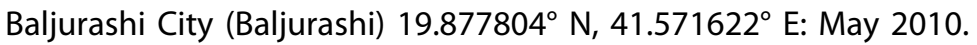

\section{Discussion}

The Arabian Peninsula is considered by many authors to include three zoogeographical regions, the Afrotropical, the Palaearctic, and the Oriental (Hölzel 1998). Many authors include the south-western part of the Arabian Peninsula in the Afrotropical Region, but there appears to be no agreement as to the extent of this region of the Peninsula (ElHawagry et al. 2013).

Al-Baha Province, like other areas in the south-western part of KSA, contains montane woodlands and evergreen shrub lands, with strong Afromontane affinities (Bussmann and Beck 1995), predominantly rich in Juniperus spp., Acacia spp., Olea spp., introduced cactus species and many other Afrotropical trees, including Nuxia oppositifolia, Maesa lanceolata and Celtis africana that grow in well-watered gullies, and mixed with some Mediterranean and northern temperate elements (Ghazanfar and Fisher 1998). 
Considering the insect fauna of Al-Baha Province as listed by El-Hawagry et al. (2013) and these additional taxa listed herein, it is concluded that Al-Baha Province has an extraordinarily complex and interesting insect fauna. This may be attributed to its geographical position at the junction of the Afrotropical Region with its Afromontane vegetation and the Palaearctic Region with its Mediterranean and temperate floristic elements (Bussmann and Beck 1995; Hölzel 1998). Sharaf, Fisher, et al. (2014) studied the ants in the south-western mountains of KSA and showed that ants in this region have considerable affinities with the Afrotropical fauna. The insect species listed in this study also support the assumption of El-Hawagry et al. (2013) and Sharaf, Fisher, et al. (2014) that the insect fauna of Al-Baha Province is strongly related to the Afrotropical Region, rather than to the Palearctic Region or the Eremic Zone. These results emphasize the fact that parts of the Arabian Peninsula, including Al-Baha Province, should be included in the Afrotropical Region rather than in the Palaearctic Region or the Eremic Zone. Other studies have confirmed the Afrotropical faunal affinities in south-western mountains of the Arabian Peninsula (Eig 1938; Bolton 1994; Aldawood et al. 2011; Sharaf and Aldawood 2011, 2012; Sharaf et al. 2012a, 2012b; Sharaf and Aldawood 2013; Sharaf, Fisher, et al. 2014).

\section{Acknowledgements}

We are grateful to Prince Bandar Bin Saud Al Saud, Head of the Saudi National Commission for Wildlife Conservation and Development for the support during the study. We are also grateful to Dr Brian Fisher, Ms Estella Ortega and Ms Michele Esposito (California Academy of Sciences, San Francisco, USA) for help in photographing the ant species Messor semirufus and Nesomyrmex denticulatus. We are indebted to the following people for providing critical assistance in various ways throughout this study: Dr Michael Engel and Dr Victor Gonzalez, University of Kansas, USA; Mr Shehzad Salman and Mr Ahmed Shams Alola, Plant Protection Department, College of Food and Agriculture Sciences, King Saud University, Saudi Arabia. Special appreciation is extended to Dr Boris C. Kondratieff, Department of Bioagricultural Sciences and Pest Management, Colorado State University, for reading the manuscript.

\section{Disclosure statement}

No potential conflict of interest was reported by the authors.

\section{Funding}

This study was supported by NSTIP strategic technology programme [project number 12ENV2484-02] in the Kingdom of Saudi Arabia.

\section{References}

Alahmed AM, Kheir SM, Al Khereiji MA. 2010. Distribution of Culicoides latreille (Diptera: Ceratopogonidae) in Saudi Arabia. J Entomol. 7:227-234.

Aldawood AS, Sharaf MR, Taylor B. 2011. First record of the myrmicine ant genus Carebara Westwood, 1840 (Hymenoptera: Formicidae) from Saudi Arabia with description of a new species, C. abuhurayri sp. n. ZooKeys. 92:61-69. 
Bolton B. 1994. Identification guide to the ant genera of the world. Cambridge (MA): Harvard University Press.

Borror DJ, Triplehorn CA, Johnson NF. 1989. An introduction to the study of insects. 6th ed. Philadelphia (PA): Saunders College Publishing.

Bussmann RW, Beck E. 1995. The forests of Mount Kenia (Kenya), a phytosociological synopsis. Phytocoenologia. 25:467-560.

Collingwood CA. 1985. Hymenoptera, Fam. Formicidae of Saudi Arabia. Fauna Saudi Arabia. 7:230-301.

Collingwood CA, Agosti D. 1996. Formicidae of Saudi Arabia (part 2). Fauna Saudi Arabia. 15:300-385.

Cranston PS, Judd DD. 1989. Diptera, Fam. Chironomidae of the Arabian Peninsula. Fauna Saudi Arabia. 10:236-289.

Crosskey RW. 1980. Catalogue of the Diptera of the Afrotropical region. London: British Museum (Natural History).

Dawah HA. 2011. Some Tachinidae (Diptera: Calyptrata) from South-Western Saudi Arabia. J Jazan Univ - Appl Sci Branch. 1:28-38.

Dawah HA, Abdullah MA. 2006. The Ephydridae (Diptera: Brachycera: Muscomorpha) of southwestern Saudi Arabia. Fauna Saudi Arabia. 21:383-394.

Dawah HA, Abdullah MA. 2008. First records of the stalk-eyed flies (Diopsidae: Cyclorrhapha), a pest of Poaceae, from South-Western Saudi Arabia. King Khalid Univ J Nat Med Tech Sci. 4:86-95.

Deeming JC, Al-Dhafer HM. 2012. Chloropidae from the Arabian Peninsula (Diptera: Cyclorrhapha). Zool Middle East. 58:3-88.

Doha SA. 2009. Phlebotomine sand flies (Diptera, Psychodidae) in different localities of Al-Baha province, Saudi Arabia. Egypt Acad J Biol Sci. 1:31-37.

Eig A. 1938. Taxonomic studies on the Oriental species of the genus Anthemis. Palest J Bot. $1: 161-224$

El-Hawagry MS, Al Dhafer HM. 2015. Five new records of bee flies (Bombyliidae, Diptera) from Saudi Arabia with zoogeographical remarks. ZooKeys. 489:125-133.

El-Hawagry MS, Khalil MW, Sharaf MR, Fadl HH, Aldawood AS. 2013. A preliminary study on the insect fauna of Al-Baha Province, Saudi Arabia, with descriptions of two new species. ZooKeys. 274:1-88.

Evans LJ. 1975. An improved aspirator (pooter) for collecting small insects. Proc Brit Entomol Nat Hist Soc Trans. 8:8-11.

Ghazanfar SA, Fisher M. 1998. Vegetation of the Arabian Peninsula. Geobotany. 25:1-362.

Greathead DJ. 1980. Diptera, Fam. Bombyliidae. Fauna Saudi Arabia. 2:291-337.

Greathead DJ. 1988. Diptera, Fam. Bombyliidae of Saudi Arabia (part 2). Fauna Saudi Arabia. 9:90-113.

Hölzel H. 1983. Insects of Saudi Arabia. Neuroptera, Fam. Ascalaphidae. Fauna Saudi Arabia. 5:235-239.

Hölzel H. 1998. Zoogeographical features of Neuroptera of the Arabian Peninsula. Acta Zool Fenn. 209:129-140.

Ibrahim AA, Abdoon MA. 2005. Distribution and Population Dynamics of Phlebotomus Sandflies (Diptera, Psychodidae) in an Endemic Area of Cutaneous leishmaniasis in Asir Region, Southwestern Saudi Arabia. J Entomol. 2:102-108.

Kirk-Spriggs AH, McGregor G. 2009. Disjunctions in the Diptera (Insecta) fauna of the Mediterranean Province and southern Africa and a discussion of biogeographical considerations. Trans R Soc S Afr. 64:32-52.

Larsen TB. 1984. Butterflies of Saudi Arabia and its neighbours. London: Stacey International.

Lelej AS, van Harten A. 2006. A review of the Mutillidae (Hymenoptera) of Yemen. Zootaxa. 1226:1-50.

Lis JA. 1999. Burrower bugs of the Old World - a catalogue (Hemiptera: Heteroptera: Cydnidae). Int J Invert Tax Genus. 10:165-249. 
Mei M, Stuke J. 2008. Remarks on Zodion nigritarsis (Strobl, 1902) and other European species of Zodion Latreille, 1796, with a revised key (Diptera, Conopidae). Tijdschr Entomol. 151:3-10.

Merz B, Dawah H. 2005. Fruit flies (Diptera, Tephritidae) from Saudi Arabia, with descriptions of a new genus and six new species. Rev Suisse Zool. 112:983-1028.

Morgulis E, Freidberg A. 2014. The Ulidiini (Diptera: Tephritoidea: Ulidiidae) of Israel, with a key to the world species of Ulidia and description of five new species. Zootaxa. 3780:201-247.

Moulet P, Kment P, Linnavuori RE, Carapezza A. 2014. Order Hemiptera, suborder Heteroptera, Infraorder Cimicomorpha, family Reduviidae. Fauna of the UAE. 5:28-91.

Pape T. 1996. A catalogue of the Sarcophagidae of the world (Insecta: Diptera). Mem Entomol Internat. 8:1-558.

Sclater PL. 1858. On the general geographical distribution of the members of the class Aves. J Proc Linn Soc Zool. 2:130-136.

Setyaningrum H, Al Dhafer HM. 2014. The Calliphoridae the blow flies (Diptera: Oestroidea) of Kingdom of Saudi Arabia. Egypt Acad J Biol Sci. 7:49-139.

Sharaf MR, Aldawood AS. 2011. Monomoriumdryhimi sp. n., a new ant species (Hymenoptera, Formicidae) of the M. monomorium group from Saudi Arabia, with a revised key to the Arabian species of the group. ZooKeys. 106:47-54.

Sharaf MR, Aldawood AS. 2012. A new ant species of the genus Tetramorium Mayr, 1855 (Hymenoptera, Formicidae) from Saudi Arabia, including a revised key to the Arabian species. PLoS ONE. 7:e30811.

Sharaf MR, Aldawood AS. 2013. First occurrence of the Monomorium hildebrandti-group (Hymenoptera: Formicidae), in the Arabian Peninsula, with description of a new species $M$. kondratieffi n. sp. Proc Entomol Soc Wash. 115:75-84.

Sharaf MR, Aldawood AS, El-Hawagry MS. 2012a. A new ant species of the genus Tapinoma (Hymenoptera, Formicidae) from Saudi Arabia with a key to the Arabian species. ZooKeys. 212:35-43.

Sharaf MR, Aldawood AS, El-Hawagry MS. 2012b. First record of the ant subfamily Aenictinae (Hymenoptera, Formicidae) from Saudi Arabia, with the description of a new species. ZooKeys. 228:39-49.

Sharaf MR, Aldhafer H, Aldawood AS. 2014. First record of the Myrmicine ant genus Meranoplus Smith, 1853 Hymenoptera: Formicidae) from the Arabian Peninsula with description of a new species and notes on the zoogeography of southwestern Kingdom Saudi Arabia. PLoS ONE. 9: e111298.

Sharaf MR, Fisher BL, Aldawood AS. 2014. Notes on ants of the genus Strumigenys F. Smith, 1860 (Hymenoptera: Formicidae) in the Arabian Peninsula, with a key to species. Sociobiology. 61:293-301.

Steyskal G. 1968. Notes \& descriptions of Egyptian acalyptrate Diptera (Ephydridae, Milichiidae, Otitidae). Bull Soc Entomol Egypte, 1966. 50:109-125.

Uvarov BP. 1938. Ecological and biogeographical relations of Eremian Acrididae. Mem Soc Biog Paris. 6:231-273.

Uvarov BP. 1952. Studies in the Arabian Orthoptera III. New genera, species and subspecies collected by the Anti-Locust Missions. Zool J Linn Soc. 42:176-194.

Wallace AR. 1876. The geographical distribution of animals. London: Mac Millan. 